\title{
L'AMSS devient ScolarMed CH
}

\author{
Annemarie Tschumper ${ }^{a}$, Ursula Kälin-Keller ${ }^{a}$, Cristina Fiorini Bernasconi ${ }^{b}$, pour ScolarMed CH \\ ${ }^{a}$ Co-Présidentes de ScolarMed $\mathrm{CH} ;{ }^{b}$ Membre du comité de ScolarMed $\mathrm{CH}$
}

\begin{abstract}
Lors de l'assemblée générale du 7 juin 2018, l’Association des Médecins Scolaires de Suisse s'est transformée en ScolarMed CH, une association interprofessionnelle ouverte à tou-te-s les professionnel-le-s de la santé travaillant dans un service de santé scolaire. ScolarMed $\mathrm{CH}$ entend continuer à soutenir l'ancrage de la médecine scolaire dans les services de santé scolaire et permettre un soutien médical adapté aux besoins de tous les élèves suisses.
\end{abstract}

Depuis plus de cent ans, les services de santé scolaire, en tant que services de santé publique reconnus, œuvrent avec les écoles afin de permettre à tous les enfants de développer leur potentiel éducatif et de promouvoir leur santé tout au long de leur scolarité.

Sous réserve des différences cantonales, les services de santé scolaires:

- contribuent à la réponse aux besoins liés à l'intégration, à l'accompagnement et à la protection des élèves en cas de maladies chroniques, absentéisme, troubles du développement, troubles du comportement, etc.;

- accompagnent les écoles dans la mise en place de projets de promotion de la santé des élèves et/ou de mesures sanitaires (hygiène, premiers secours);

- luttent contre les maladies infectieuses, contrôlent les carnets de vaccination conformément à la loi sur les épidémies, et proposent des vaccinations scolaires (p. ex. HPV);

- effectuent des examens médicaux, avec la possibilité de signaler et de prendre en charge les élèves ayant besoin d'un suivi.

L'activité des services de santé scolaire se veut complémentaire à la prise en charge des médecins traitants (avec qui les services de santé collaborent) et prend en compte la santé individuelle et collective des élèves. Grâce à leur proximité avec l'école et le système de soins médicaux, les services de santé scolaire jouent souvent un rôle de pont entre les soignant-e-s, l'école, les élèves et leur famille. Ils garantissent ainsi la mise en place de mesures médicales individuelles adaptées visant à soutenir l'apprentissage scolaire. Plusieurs services recueillent et analysent régulièrement les données de santé des élèves en les rendant ano- nymes, afin d'orienter leurs activités et d'organiser des programmes communautaires de promotion de la santé.

Différent-e-s professionnel-le-s font partie des services de santé scolaire:

- Les médecins scolaires sont responsables de la prise en charge de la santé des élèves dans le cadre du service de santé scolaire. Ils sont à disposition des écoles et des parents pour répondre aux questions en lien avec la santé des élèves, effectuer des examens médicaux, des vaccinations dans le cadre des prescriptions cantonales et sont garant de la situation sanitaire dans les écoles (hygiène, organisation des mesures d'urgence, etc.).

- Les infirmier-e-s scolaires, surtout en Suisse romande, mais de plus en plus en Suisse alémanique, sont les personnes de référence pour la santé des élèves à l'école, organisent la vaccination, les dépistages et des entretiens médicaux en étroite collaboration avec les médecins scolaires et collaborent à la mise en place de projets de promotion de santé.

- Les assistant-e-s médical-e-s ont une bonne connaissance de l'organisation de l'école et contribuent à établir un lien de confiance entre l'école, la famille et le service de santé scolaire. Avec une formation appropriée, les assistant-e-s médical-e-s accomplissent des actes médicaux délégués tels que dépistages ou consultations simples.

Les professionnel-le-s des services de santé scolaire constituent sans aucun doute le plus grand effectif de santé publique en Suisse, dont l'association professionnelle ScholarMed $\mathrm{CH}$ peut se prévaloir de plus de 100 ans d'histoire. Elle est issue de «l'association des 
soins de santé scolaire» fondée en 1899, qui a donné vie en 2005, après plusieurs étapes et fusions avec d'autres organisations, à Santé Publique Suisse avec qui ScolarMed CH est associée.

C'est ainsi que ce passage d'une association purement médicale à une association interprofessionnelle, est un pas en avant qui prend en compte la dimension interprofessionnelle de la médecine scolaire actuelle au sein des services de santé scolaire.

L'association ScolarMed CH s'engage:

- pour la santé des élèves et l'accès à des soins médicaux scolaires spécialisés pour tous les élèves;

- pour des prestations de médecine scolaire de qualité: Le travail en santé scolaire exige des connaissances dans différents domaines qu'ils soient médicaux (pédiatrie, vaccinologie, infectiologie), pédagogiques (plan d'études, organisation scolaire) ou de santé publique, de médecine du travail ou des approches de promotion et prévention de la santé. Avec l'offre de formation continue en collaboration avec des universités et des hautes écoles, une page web www. scolarmed.ch avec accès à des contributions techniques approfondies pour les membres et l'établissement de documents de référence selon la règle de l'art, ScolarMed $\mathrm{CH}$ entend contribuer au maintien de la qualité des prestations;

- dans la promotion de la coopération et des échanges entre professionnel-le-s, ainsi que de l'identité professionnelle de ses membres.

ScolarMed CH se réjouit d'accueillir de nombreux nouveaux membres parmi les médecins et infirmier-e-s scolaires, ainsi que des assistant-e-s médical-e-s de tous les cantons.

ScolarMed CH offre à ses membres: l'accès à de nombreuses informations techniques et pratiques sur la partie protégée du site web de ScolarMed CH, la possibilité de contacter les collègues via la base de données des membres, la possibilité de poser toute question ou préoccupation au comité de ScolarMed CH, ainsi qu'une information régulière concernant les formations de médecine scolaire et d'autres actualités et nouveautés. ScolarMed $\mathrm{CH}$ invite également chaque professionnel-le intéressé à participer à la prochaine réunion nationale, qui aura lieu à Saint-Gall le 28 november 2019. Le comité de l'association se tient à disposition pour tout renseignement sur ScolarMed $\mathrm{CH}$. Des renseignements sont aussi disponibles sur le site internet de ScolarMed CH: www.scolarmed.ch 